\title{
Influence of Whole Body Vibration and Specific Warm-ups on Force during an Isometric Mid-Thigh Pull
}

\author{
Vanessa L. Cazás-Moreno (Corresponding author) \\ School of Applied Sciences, University of Mississippi, 215 Turner Center, P.O. Box 1848 University, MS 38677-1848 \\ E-mail: vlcazas@olemiss.edu \\ Jacob R. Gdovin \\ School of Applied Sciences, University of Mississippi, 215 Turner Center, P.O. Box 1848 University, MS 38677-1848 \\ E-mail: jrgdovin@olemiss.edu \\ Charles C. Williams \\ School of Applied Sciences, University of Mississippi, 215 Turner Center, P.O. Box 1848 University, MS 38677-1848 \\ E-mail: ccwilli1@olemiss.edu \\ Charles R. Allen \\ School of Applied Sciences, University of Mississippi, 215 Turner Center, P.O. Box 1848 University, MS 38677-1848 \\ E-mail: crallen@olemiss.edu \\ Yang-Chieh Fu \\ School of Applied Sciences, University of Mississippi, 215 Turner Center, P.O. Box 1848 University, MS 38677-1848 \\ E-mail: ycfu@olemiss.edu \\ Lee E. Brown \\ College of Health and Human Development, California State University, Fullerton, 800 North State College Blvd., Fullerton, CA 92834 \\ E-mail: leebrown@fullerton.edu \\ John C. Garner III \\ School of Applied Sciences, University of Mississippi, 215 Turner Center, P.O. Box 1848 University, MS 38677-1848 \\ E-mail: jcgarner@olemiss.edu
}

Received: 02-09- 2015

Accepted: 10-10- 2015

Published: 31-10- 2015

doi:10.7575/aiac.ijkss.v.3n.4p.31

URL: http://dx.doi.org/10.7575/aiac.ijkss.v.3n.4p.31

\begin{abstract}
Purpose: The purpose of this study was to investigate the effects of general and specific warm-up protocols on rate of force development (RFD), relative RFD (rRFD), ground reaction force (GRF) and relative ground reaction force (rGRF) during an isometric mid-thigh pull (IMTP), after WBV exposure. Methods: Fifteen healthy recreationally trained males (age: $24.1 \pm 2.3 \mathrm{yrs}$, height: $72.9 \pm 7.8 \mathrm{~cm}$; mass: $86.9 \pm 8.3$ ) completed five protocols: baseline, isometric vibration (iVib), isometric no vibration (iNV), dynamic vibration (dVib) and dynamic no vibration (dNV). The baseline was completed without any warm-up prior to the IMTP. The intervention protocols had the same prescription of 4 sets of 30 -second bouts of quarter squats (dynamic [DQS] and isometric [IQS]) on the WBV platform with or without vibration. Following a one-minute rest period after each protocol, participants completed three maximal IMTPs. Results: Repeated measures ANOVA with a Bonferroni post hoc demonstrated that RFD in dNV (7657.8 \pm 2292.5 N/s) was significantly greater than $\mathrm{iVib}(7156.4 \pm 2170.0 \mathrm{~N} / \mathrm{s})$. However, the other experimental trials for RFD demonstrated no significant differences $(\mathrm{p}>0.05)$. There were also no significant differences for $\mathrm{rRFD}$, GRF or rGRF between protocols. Conclusion: These results demonstrate that a dynamic warm-up without WBV elicits greater RFD than an isometric warm-up with WBV prior to a maximal isometric exercise. Further research needs to be investigated utilizing dynamic and isometric warm-ups in conjunction with WBV and power output.
\end{abstract}

Keywords: males, recreationally trained, power

\section{Introduction}

Improvements in power, developed from multiple training sessions, have been shown to enhance sport performance (Adams et al., 2009; Gerakaki et al., 2013; Haff et al., 2005; Kurt et al., 2014; Lamont et al., 2010). There are a number of traditional methods such as anaerobic and aerobic protocols that are utilized which lead to an increase in power production; however, athletes and coaches alike are continually looking for novel methods that are more efficient at 
improving performance (Cardinale \& Bosco, 2003; Chmielewska et al., 2014; Dabbs et al., 2010; Dabbs et al., 2011; Dabbs et al., 2012; Dallas et al., 2014; Kurt et al., 2014; Lamont et al., 2010; Marin et al., 2013; Rittweger et al., 2000; Ritzmann et al., 2013; Ronnestad et al.,2012). Traditionally, a warm-up is recommended prior to performing an exercise (Thompson et al., 2013). Warm-ups typically consist of both general and specific activities. The general warm-up consists of full body movements that are used to increase the body's temperature, blood flow, functional mobility, and muscle activation (i.e. jogging or jumping jacks) (Thompson et al., 2013). The specific warm-up consists of movements directly related to the performance related tasks (i.e. mimicking movements utilized during performance) (Thompson et al., 2013).

Participating in a specific warm-up prior to a vigorous activity has been shown to enhance lower body strength and power (Alikhajeh et al.,2011; Hamada et al., 2000; Ribeiro et al., 2014). However, if the intensity of the specific warmup is too low, it may not augment performance (Anderson, et al., 2014; Ribeiro et al., 2014). Incorporating a supplementary, specific warm-up, may lead to increased motor unit recruitment and synchronization (Gerakaki et al., 2013; Lamont et al., 2010). It has been demonstrated that an increase in performance may occur as a result of applying a specific warm-up that utilizes dynamic and isometric exercises that are aimed at eliciting an increase in force production (Lamont et al., 2010). In addition to dynamic and isometric exercises, whole body vibration (WBV) may be an alternative warm-up method prior to an explosive bout of exercise. In an acute study conducted by Holt et al. (2008), they demonstrated the use of a dynamic specific warm-up resulted in an increased vertical jump performance in comparison to a general warm-up and stretching protocol. Similarly, a study by Thompsen et al. demonstrated that both horizontal and vertical jumps were enhanced in female collegiate athletes after utilizing a body weight dynamic warmup in comparison to a static or weighted warm-up. Frantz et al. ( 2011), demonstrated that collegiate baseball players had significant enhancements in lower body power after utilizing body weight exercise dynamic warm-up in comparison to a static stretching warm-up. Anderson et al. (Anderson et al., 2014) found that high intensity warm-ups elevated trained individuals' heart rate to near maximal intensity, and this significantly increased high intensity sprint intervals. These results indicate that the use of a dynamic specific warm-up may be beneficial for trained individuals prior to an acute bout of power exercises.

The purpose of warm-ups are to prepare an athlete for competition (Thompson et al., 2013). However, some warm-ups have failed to increase lower body performance (Anderson et al., 2014; Cardinale \& Bosco, 2003; Reiman et al., 2010; Ribeiro et al., 2014). Ribeiro et al. (2014) reported that the effects of three different warm-ups did not enhance the number of repetitions to failure during a submaximal squat exercise in recreationally trained individuals'. Aerobic, anaerobic, and stretching exercises did not influence vertical jump performance according to Christensen et al. (2008) Furthermore, Reiman et al. (2010) revealed that teenage athletes did not demonstrate significant differences in lower body power when warming-up with a weighted vest that was $5 \%$ of their body weight in comparison to a body weight dynamic protocol. These results indicate that the intensity of the warm-up activities may have been too fatiguing or there was not an adequate amount of rest in between the warm-up and the explosive activity.

Whole-body vibration has been implemented as a specific warm-up alone and in conjunction with dynamic exercises (i.e. squats) prior to explosive activity (Cardinale \& Bosco, 2003; Chmielewska et al., 2014; Dabbs et al., 2010; Dabbs et al., 2011; Dabbs et al., 2012; Dallas et al., 2014; Kurt et al., 2014; Lamont et al., 2010; Marin et al., 2013; Rittweger et al., 2000; Ritzmann et al., 2013; Ronnestad et al., 2012; Turner, 2011). Dallas et al. ( 2014) utilized a low vibration frequency of $30 \mathrm{~Hz}$ prior to vertical jump performance and found that jump height significantly increased. Similarly, Dabbs et al. (2010) demonstrated that maximum jump height also significantly improved after WBV exposure at $30 \mathrm{~Hz}$. In another study, jump height significantly increased at a higher frequency of $40 \mathrm{~Hz}$ (Turner et al., 2011). This demonstrates that utilizing WBV as a specific warm-up, may lead to acute increased neuromuscular performance (force and power) prior to an explosive bout of exercise (Cardinale \& Bosco, 2003; Dabbs et al., 2010; Dabbs et al., 2011; Dabbs et al., 2012; Dallas et al., 2014; Marin et al., 2013; Rittweger et al., 2000; Ritzmann et al., 2013; Ronnestad et al., 2012).

Conversely, the literature has also shown that WBV has failed to demonstrate significant increases in performance (Chmielewska et al., 2014; Rittweger et al., 2000; Ritzmann et al., 2013). Chmielewska (2014) demonstrated that WBV did not alter motor unit recruitment of the rectus femoris after exposure to either low- or high- frequency vibration during repeated squats. Similarly, in an applied setting, Rittweger et al. (2000) indicated that acute explosive power decreased after WBV exposure. Furthermore, Ritzmann (2013) reported that the H-reflex was negatively altered following WBV which led to a lack of performance enhancement. As debates continues in the literature regarding WBV, it is important to note that its effects may be highly individualized (Chmielewska et al., 2014) indicating the time to enhancement may be individualized.

An isometric mid-thigh pull (IMTP) is an explosive and complimentary exercise to the Olympic lifts. The IMTP manipulates the start of the second pull of the clean exercise and attempts to reach triple extension of the ankles, knees and hips (Comfort et al., 2011; Jones et al., 2015; Haff et al., 2005). An IMTP requires maximal effort, yet the exercise is isometric and joint angles do not change from the starting hang position. The IMTP has been used in research settings to measure applied forces and has also been used as a training tool to possibly eliminate poor technique (Haff et al., 2005). There have been a number of studies that have investigated RFD and GRF during an IMTP (Comfort et al., 2011; Haff et al., 2005; Haff et al., 2015; Khamoui et al., 2011) with Haff et al., (2015) demonstrating that the IMTP is an acceptable and reliable exercise. 
To date, there are no standardized warm-up protocols prior to a maximal isometric power output. The literature reflects multiple combinations of time and exercise methods utilizing static, dynamic and WBV (Adams et al., 2009; Chmielewska et al., 2014; Dabbs et al., 2010; Dabbs et al., 2011; Dabbs et al., 2012; Dallas et al., 2014; García-López et al., 2012; Gerakaki et al., 2013; Kurt et al., 2014; Lamont et al., 2010; Marin et al., 2013; Rittweger et al., 2000; Ronnestad et al., 2012; Turner et al., 2011) making it important to further examine its applicability to improvements in the rate of force development (RFD), relative RFD (rRFD), ground reaction force (GRF) and relative ground reaction force (rGRF) during sport performance after warm-up exposure (Alikhajeh et al., 2011; Chmielewska et al., 2014; Cilli et al., 2014; Dabbs et al., 2010; Dabbs et al., 2011; Dallas et al., 2014; García-López et al., 2012; Marin et al., 2013; Rittweger et al., 2000; Ritzmann et al., 2013; Ronnestad et al., 2012; Thompson et al., 2013; Turner et al., 2011). As previously mentioned, utilizing a specific warm-up is highly recommended prior to any maximal effort lower body power activity. It has been shown (Lamont et al., 2010) that the use of WBV as a specific warm-up resulted in an acute increase in power and force production during a maximal isometric muscle action. Further investigation is needed to determine the effects of a dynamic, isometric and combination mode of warm-up prior to a maximal isometric muscle action. It is plausible that WBV in conjunction with a specific warm-up may lead to an increase in acute power and force production during an IMTP. Furthermore, research has demonstrated that there is a significant correlation between IMTP and dynamic and explosive exercises (Haff et al., 2005; Khamoui et al., 2011; Marin et al., 2013). The IMTP exercise was selected for this study to minimize the variance between participants, isolate the dependent variables of interest (Comfort et al., 2011) and test the applicability of the findings (Haff et al., 2015; Lamont et al., 2010). Therefore, the purpose of this study was to determine the influence of isometric and dynamic exercises in conjunction of WBV versus no WBV on the RFD, rRFD, GRF and rGRF during an IMTP.

\section{Methods}

Each participant completed one-familiarization, a baseline and four intervention sessions separated by a minimum of one hour. Three maximal IMTP's were performed one minute after each intervention session (specific warm-up) was completed. Therefore, a within subject repeated measures study design was used to examine RFD, rRFD, GRF and rGRF.

\subsection{Participants}

Fifteen healthy, recreationally trained males who were free of any lower body orthopedic and/or musculoskeletal injuries completed the study (age: $24.1 \pm 2.3 \mathrm{yrs}$, height: $72.9 \pm 7.8 \mathrm{~cm}$; mass: $86.9 \pm 8.3$ ). Recreationally trained was defined as completing a minimum of three hours per week of resistance training over the last year prior to the initiation of the study. All participants read and signed a University Institutional Review Board (IRB) approved informed consent.

\subsection{Procedures}

Each participant was asked to avoid any lower body aerobic or anaerobic training 24 hours prior to the start and for the duration of the study. During the familiarization session, participants had their anthropometrics recorded, followed by verbal instructions and visual demonstration of the general warm-up and the IMTP. Three non-recorded, self-selected effort pulls of the IMTP were completed at this time. Participants were instructed to pull at minimal to moderate effort. Following this, each participant was given verbal instructions and a demonstration of how to perform the isometric quarter squat (IQS) and the dynamic quarter squats (DQS) during vibration and no vibration exposure while standing on the WBV platform. As participants stood on the vibration platform, IQS were completed simultaneously until they felt comfortable with the task; DQS were completed after the isometric condition. For the familiarization without vibration, participants simply stood on the platform while performing both quarter squat conditions. Participants were instructed to wear the same footwear throughout the remainder of the study.

During the first testing session, baseline testing of the IMTP was conducted prior to any warm-up. The baseline test consisted of three maximal IMTP on the modified Jones Machine (BODYCRAFT, Lewis Center, OH) (Figure 1). Participants were given the following instructions prior to each maximal IMTP: "pull as hard as you can, as fast as you can, while driving your feet down into the ground". Encouragement was given during each pull. The IMTP was held for 3 -seconds and a 15-second rest period was given between each maximal pull. Lifting straps were worn for all pulls.

After baseline testing, each participant performed a general warm-up and completed the following protocols based on a counterbalanced design: IQS with vibration (iVib), IQS with no vibration (iNV), DQS with vibration (dVib), and DQS with no vibration (dNV). After completing the specific warm-up, one-minute of rest was given prior to the post-test (Cazas et al., 2013). Post-testing utilized the same protocol as baseline testing. This procedure was repeated until each participant completed each specific warm-up protocol. Each condition was separated by a minimum of one hour. 2.3 Warm Up Descriptions

The general warm-up was completed prior to each specific warm-up protocol and consisted of 10 bodyweight squats, 10 $\mathrm{m}$ of walking knee hugs and $10 \mathrm{~m}$ Frankenstein marches. During the walking knee hugs participants stepped forward with one foot and went into full range of motion of plantar-flexion, as the opposite leg flexed at the knee and the hip while the participant pulled their knee into their chest simultaneously. Frankenstein marches consisted of stepping forward with one foot and kicking the opposite leg into the air at maximal range of motion, while maintaining straight legs. After their perceived maximum range of motion had been achieved, the kicking leg returned to starting position and then switched legs. 
The specific warm-up consisted of 4 sets of 30-second bouts in conjunction with the following: IQS with vibration. (iVib), IQS with no vibration (iNV), DQS with vibration (dVib), DQS with no vibration (dNV) while standing on the vibration plate. Thirty-seconds of rest was given between each bout. All vibration conditions were completed at $30 \mathrm{~Hz}$ and the no vibration protocols were completed at $0 \mathrm{~Hz}$ while standing on the vibration platform. During the 30 -second bouts, participants completed one of the intervention conditions at a time. The IQS was defined as a muscle action that did not alter any changes in the joint angles, thus maintaining a constant musculotendinous length for the duration of the 30-second bout (Garner, 2008). The DQS was defined as repetitive eccentric to concentric muscle actions at a 1 to 1 ratio. The researcher monitored the IQS and the 1-second to 1-second ratio for each participant. 2.4 Isometric Mid-thigh Pull

A standard Olympic barbell and a modified Jones Machine were used to perform the IMTP. The Jones Machine hooks had been reversed, so the barbell could not move when an external load was placed on it. The barbell was held directly under the hooks by straps (Figure 1). To mimic a power stance, participants started in a hang position between 120- and 130-degree knee flexion (West et al., 2011). Hip flexion was maintained in a comfortable position in response to the knee flexion throughout the exercise. By placing the participant in an optimal force-generating angle, the power stance may potentially result in the greatest amount of power production (Comfort et al., 2011; Haff et al., 2015; Kawamori et al., 2006). The barbell placement was adjusted with respect to the height of each participant; a goniometer was used to measure the knee joint angle. Hip flexion was in a fixed angle relative to the comfort of the participant. Foot placement was slightly greater than shoulder width while an overhand grip placement was used on the barbell; this grip position was directly on the lateral sides of the participant's quadriceps. Prior to initiation of the exercise, participants set up in the power position with their weight distributed evenly on both feet. A certified strength and conditioning specialist (CSCS) monitored all participants to ensure safety and proper technique was performed. Proper technique consisted of maintaining a flat back, head placement in a neutral position, and shoulders positioned slightly over the bar. To initiate the action, participants were instructed to explosively drive through their feet, in an attempt to extend at the ankles, knees and hips. For this study, the IMTP was defined as a maximal concentric loading exercise, yet there were no changes in joint angle (Garner et al., 2008). This resulted in a concentrically loaded, maximal isometric muscle action, where no mechanical movement occurred.

\subsection{Equipment}

The force plate was a $0.6 \mathrm{~m} \times 0.4 \mathrm{~m}$ force platform (Bertec Corporation, Columbus, $\mathrm{OH}$, Inc.) embedded in floor and was used to measure IMTP RFD and GRF with a sampling rate of $1000 \mathrm{~Hz}$.

The Power Plate pro5 (Northbrook, IL), which had a $86 \mathrm{~cm}$ x $94 \mathrm{~cm}$ (W x D) vibration platform was used. It administered a pivotal vibration frequency at $30 \mathrm{~Hz}$ with a vertical amplitude of 2-4 $\mathrm{mm}$.

\subsection{Data Analysis}

Data analysis was conducted by a self-developed code written in Matlab (Mathworks, Inc., Natick, MA, USA). The interval of movement during the analysis was from the time point of when the vertical ground reaction force (VGRF)

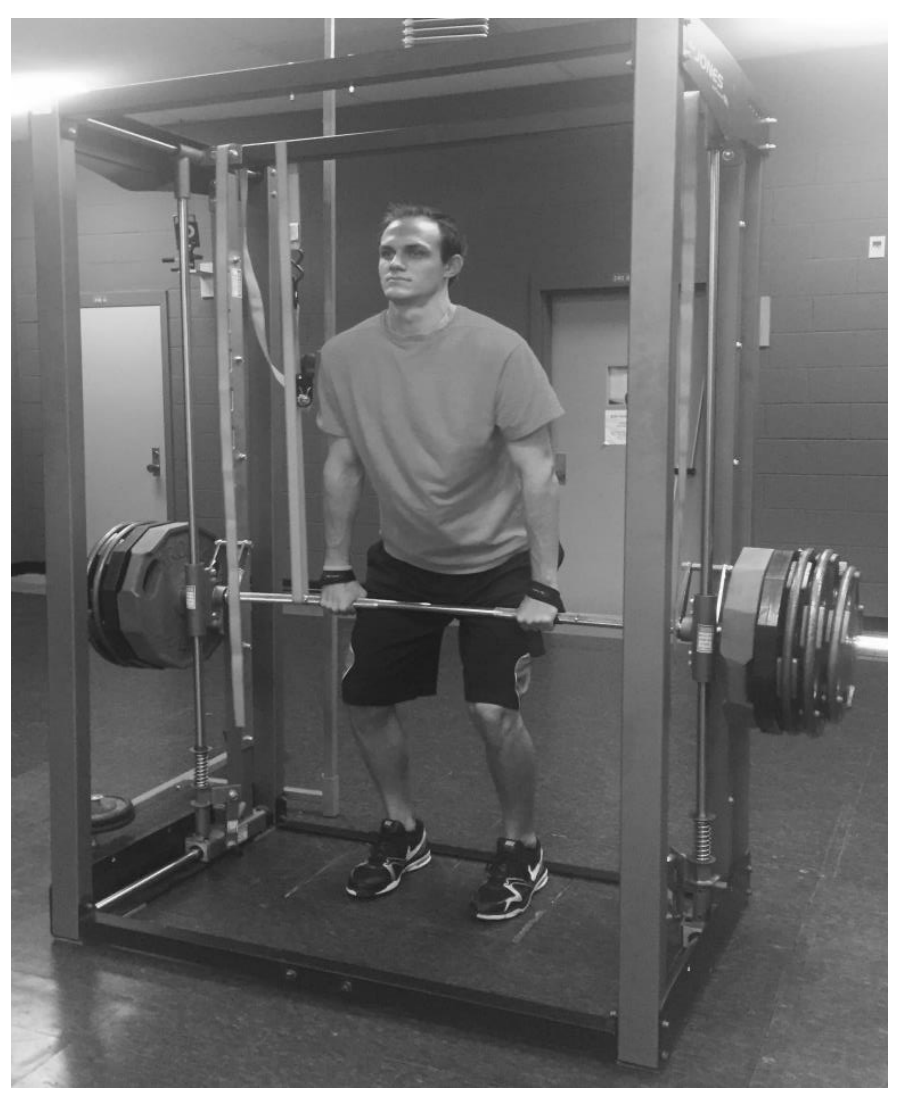

returned back to the participant's body mass to the end of the pull (see figure 1). RFD was defined by the amount of force produced over the change in time $(200 \mathrm{Nm} / \mathrm{s})$ initiated at the beginning of the movement interval (Haff et al., 2015; Lamont et al., 2010). Relative RFD was defined as the absolute RFD divided by the participant's bodyweight in kilograms $(\mathrm{Nm} / \mathrm{s} / \mathrm{kg})$. GRF was defined as the maximum VGRF throughout the entire movement interval. GRF was analyzed at the time point at which the participants force peaked throughout the pull. Relative GRF was defined as the absolute GRF divided by the participant's bodyweight in kilograms ( $\mathrm{N} / \mathrm{kg})$. Multiple 1 × 5 repeated measures analysis of variance (ANOVA) were used to determine differences between conditions. All statistical procedures were conducted using SPSS (version 22, Chicago, IL, USA). An a priori alpha level was set at 0.05 . A Bonferroni post hoc was used to highlight any between condition differences.

Figure 1. Isometric Mid-Thigh Pul

Description: the barbell was securely fixed with straps, while the weight added to the barbell was added to ensure the Jones Machine would not move. 


\section{Results}

There was a significant main effect for RFD. Post hoc testing revealed dNV was significantly greater than iVIB with an overall effect size of .84 . No other conditions were different (figure 2). There were no significant ( $p>0.05)$ differences between conditions for rRFD, GRF or rGRF (table 1). Individual percent change from baseline for GRF, rGRF, RFD and rRFD are illustrated for each condition in figure 3, 4, 5 and 6 , respectively.

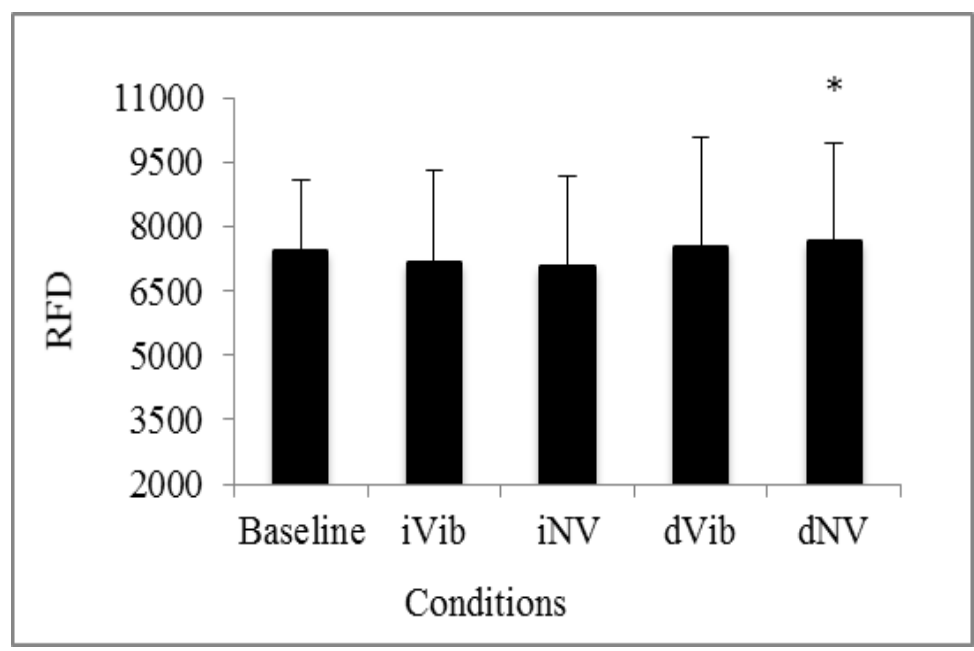

Figure 2. Rate of Force Development

Description: Maximum RFD values for all five conditions: isometric vibration (iVib); isometric no vibration (iNV); dynamic vibration (dVib); and dynamic no vibration (dNV); Group means: mean $\pm \mathrm{SD} ;(\mathrm{Nm} / \mathrm{s})$; * significantly greater than iVib.

Table 1. Mean $\pm \mathrm{SD}$ for relative RFD (Nm/s/kg), GRF (N) and relative GRF (N/kg) for all five conditions: isometric vibration (iVib); isometric no vibration (iNV); dynamic vibration (dVib); and dynamic no vibration $(\mathrm{dNV})$

\begin{tabular}{lcccc}
\hline \multicolumn{1}{c}{ Variable } & Baseline & iVib & iNV & dVib \\
\hline rRFD & $85.31 \pm 17.38$ & $82.77 \pm 24.72$ & $81.53 \pm 22.87$ & $87.14 \pm 29.08$ \\
\hline GRF & $3132.6 \pm 509.5$ & $3124.4 \pm 598.6$ & $3083.9 \pm 496.7$ & $3134.7 \pm 509$ \\
\hline rGRF & $36.09 \pm 5.64$ & $36.11 \pm 6.77$ & $35.62 \pm 5.32$ & $36.31 \pm 6.25$ \\
\hline
\end{tabular}

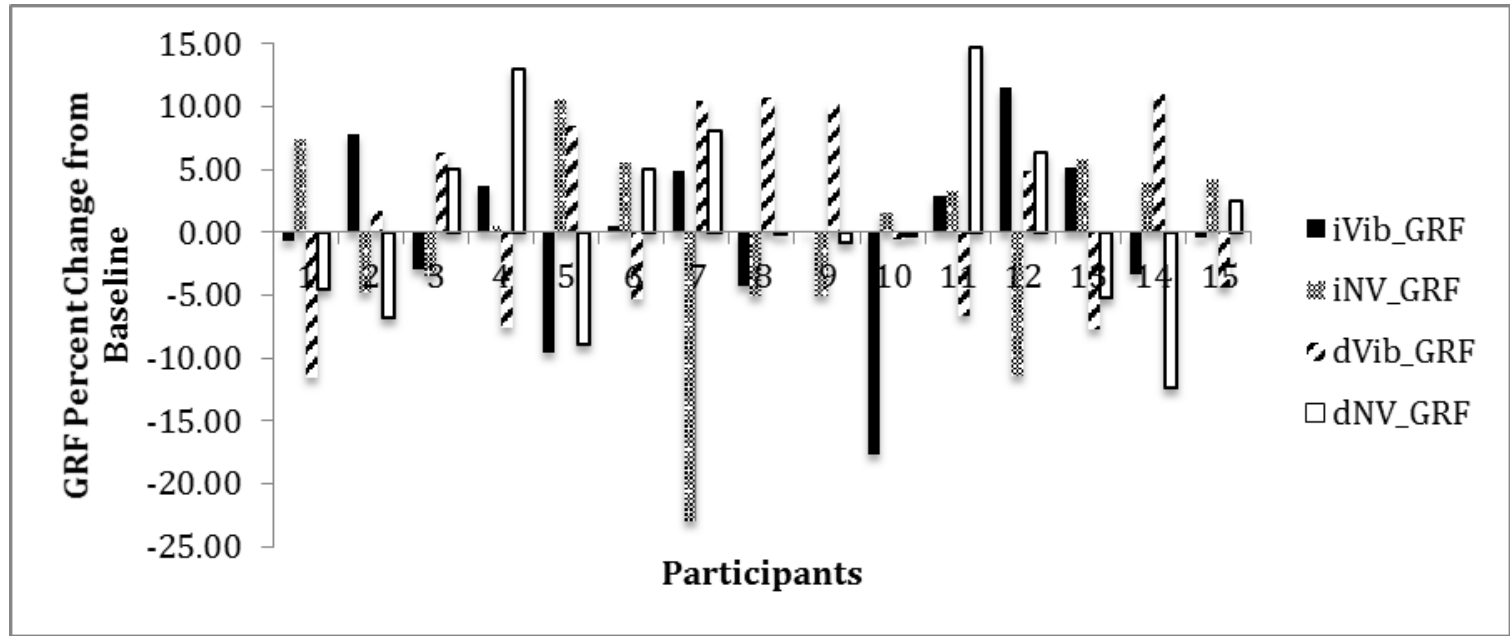

Figure 3. Ground Reaction Force Percent Change from Baseline.

Description: GRF percent change from baseline for each participant and condition. 


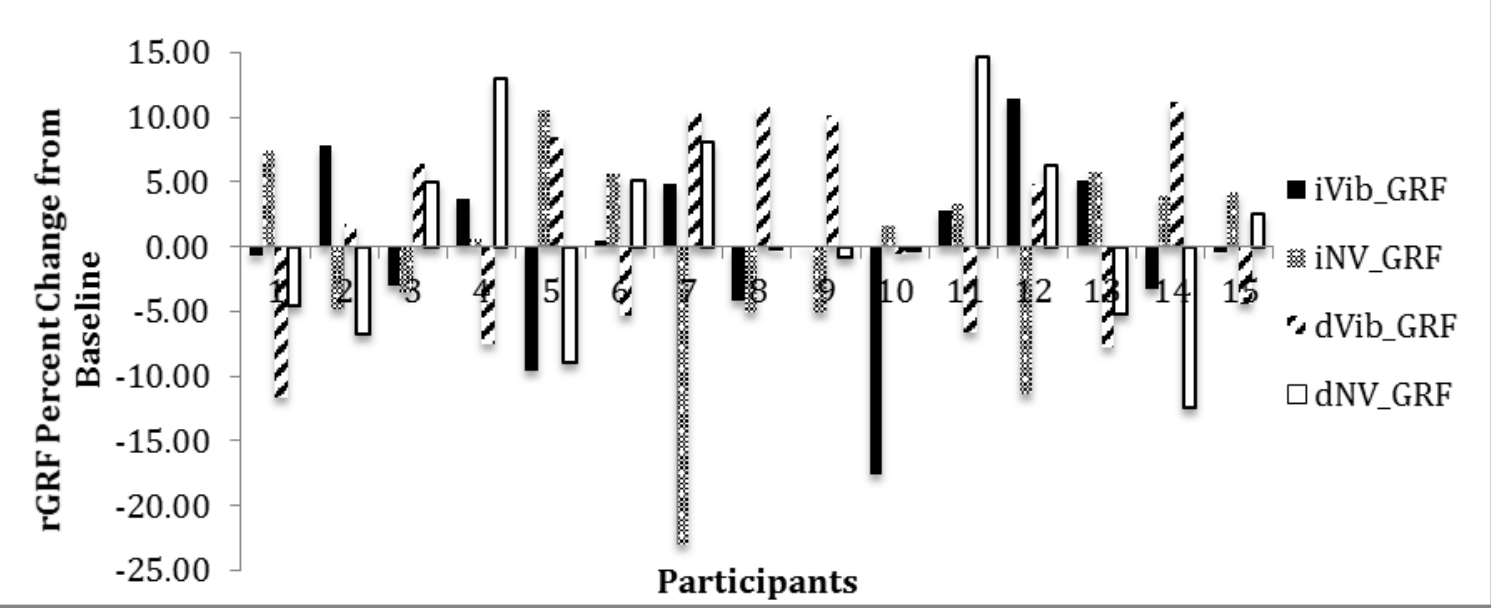

Figure 4. Relative Ground Reaction Force Percent Change from Baseline.

Description: rGRF percent change from baseline for each participant and condition.

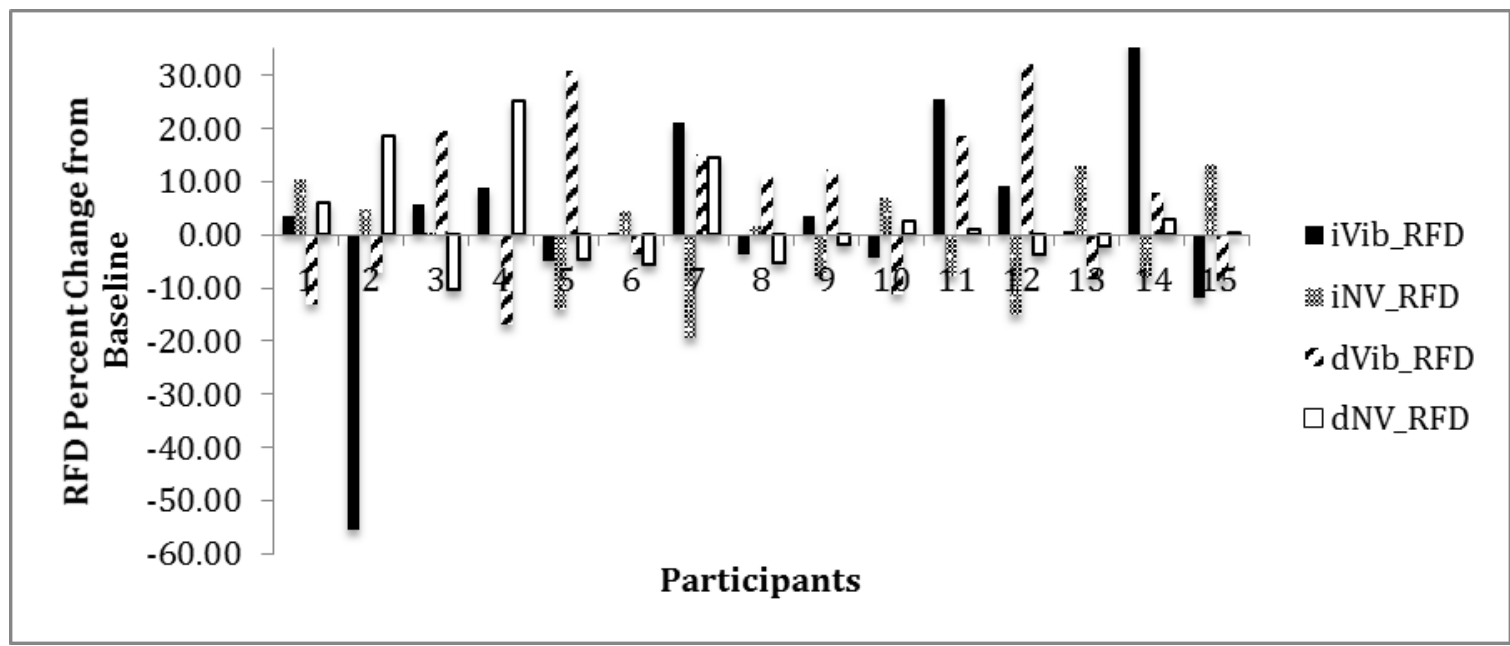

Figure 5. Rate of Force Development Percent Change from Baseline.

Description: RFD percent change from baseline for each participant and condition.

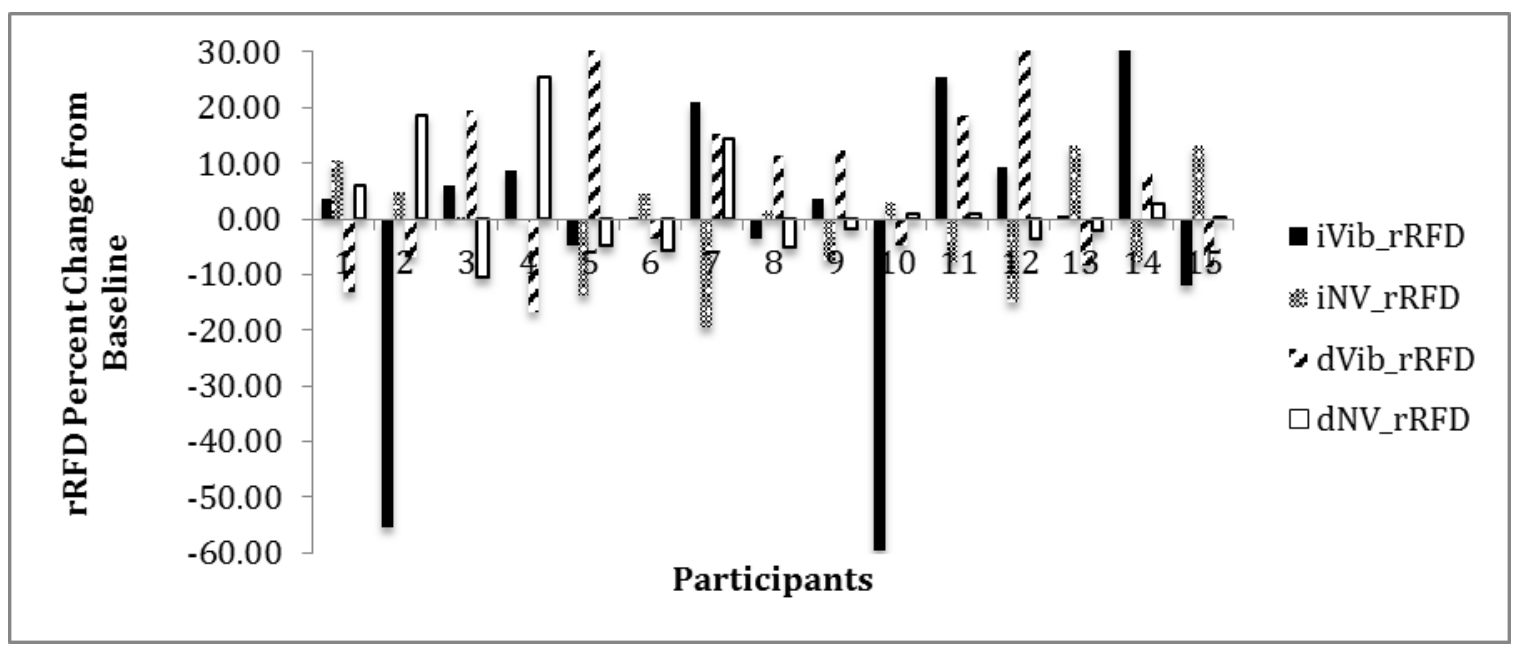

Figure 6. Relative Rate of Force Development Percent Change from Baseline.

Description: rRFD percent change for each participant and condition.

\section{Discussion}

The purpose of this study was to investigate the influence of dynamic and isometric specific warm-ups in conjunction with WBV on acute GRF and RFD during an IMTP. The main finding of this study was that no warm-up condition demonstrated any significant differences from baseline. A secondary finding was that the dynamic, specific warm-up without WBV elicited greater absolute RFD in comparison to the isometric, specific warm-up with WBV, but was not 
different from any other condition. To our knowledge, this is the first study to investigate the effect of WBV in conjunction with different specific warm-ups on RFD, and GRF during an IMTP.

Currently, there is a lack of research investigating WBV in conjunction with dynamic and isometric specific warm-ups on an isometric exercise. Therefore, it is difficult to directly compare current literature to the present study. However, the design of this study was based off WBV, resistance training and resistance exercise protocols (Adams et al., 2009; Chmielewska et al., 2014; Dabbs et al., 2010; Dabbs et al., 2011; Dabbs et al., 2012; Dallas et al., 2014; Gerakaki et al., 2013; Kurt et al., 2014; Lamont et al., 2010; Marin et al., 2013; Rittweger et al., 2000). As WBV has been shown to prime the higher order motor units prior to an explosive exercise.

There are a number of variables that influence the ability of a warm-up to enhance performance, such as intensity, load, training age, biological age, and fiber type (Alikhajeh et al., 2011). The present study used recreationally trained individuals to investigate lower body isometric strength after various warm-up protocols. Berning et al. (2010), reported that a general warm-up (5-minutes of cycling) in conjunction with one, 3-second heavy isometric squat (150\% of the participants 1RM) elicited greater vertical jump height in recreationally trained individuals indicating that a supramaximal isometric muscle action enhances performance. This suggests the isometric intensity utilized in the present study may not have been great enough to elicit increased strength. Thompsen et al. (2007) investigated the influence of a weighted specific warm-up in comparison to a body weight specific warm-up in division III athletes. They reported that vertical and horizontal jump distance was enhanced following a dynamic warm-up in comparison to a static warm-up protocol. Similarly, Lowery et al. (2012) investigating the effects of low (56\%), moderate (70\%) and high intensity (93\%) warm-ups on vertical jump performance, and showed that low intensity did not enhance performance. However, with an adequate amount of recovery ( $\sim 4$ minutes), moderate and high intensity increased performance. Similarly, Frantz et al. (2011) demonstrated that a dynamic warm-up consisting of body weight exercises stimulated an increase in vertical jump height and horizontal jump distance, but horizontal jump distance decreased after an isometric warm-up compared to no warm-up. This suggests that a low intensity isometric warm-up prior to an explosive power exercise may not enhance or even reduce performance. In contrast, the present study demonstrated no significant negative effects on performance following an isometric warm-up.

Lamont et al. (2010) compared isometric quarter squat training with a combination of WBV and resistance training versus resistance training alone and demonstrated that neither post-training GRF or RFD at $250 \mathrm{~ms}$ were significantly different from baseline measures. Their results are similar to the present study in that there were no significant differences between groups for GRF. This suggests that WBV in conjunction with isometric exercises could be utilized as a general or specific warm-up. An acute investigation by Bush et al. (2015) analyzed dynamic and isometric warmups with and without WBV in untrained individuals on knee extension strength. They demonstrated that there were significant increases in force production after the dynamic vibration protocol, but a significant decrease for all other conditions (dynamic and isometric without vibration and isometric with vibration). Furthermore, it was indicated that the decline in force production occurred during post-testing and was a result of fatigue (Bush et al., 2015). In the present study, none of the conditions elicited a significant difference in force production. This suggests that untrained individuals may demonstrate acute force increases following dynamic squats with WBV, while resistance trained individuals may require increased volume or intensity. Additionally, as the dynamic protocol used by Bush et al. (2015) had a longer eccentric to concentric ratio for dynamic squats, this and the 1- to 1-second ratio used in the present study may support why a decrease in the applied force output was not seen presently.

WBV has demonstrated that it may elicit improvements in acute force and power performance in resistance trained individuals (Adams et al., 2009; Cormie et al., 2006; Dabbs et al., 2010; Dabbs et al., 2011; Dabbs et al., 2012; Ronnestad et al., 2012). It has been hypothesized to activate the alpha-motor neurons, which may improve performance measures in an acute bout of explosive performance during an isometric muscle action (Lamont et al., 2010). In theory, the combination of WBV with dynamic or isometric resistance exercises may alter the neuromuscular contractile activity, leading to an increase in applied force production by recruiting higher order motor units. An acute study conducted by Cormie et al. (2006) found that jump height was significantly increased following WBV at $30 \mathrm{~Hz}$ when performing an isometric squat. This may infer that the combination of WBV and an isometric warm-up may increase applied force prior to a dynamic movement questioning the rule of specificity. Ronnestad et al. (2012) reported an increase in acute peak power in an explosive dynamic exercise after a bout of WBV at $50 \mathrm{~Hz}$ with no significant differences in dynamic strength in comparison to no WBV . In contrast to the present study, explosive exercise elicited a significant increase RFD after a dynamic protocol without vibration in comparison to the isometric warm-up with vibration. This may indicate that the total volume or intensity of WBV may have been too low in the present study to see an increase in IMTP performance.

\section{Conclusion}

These results suggest that WBV consisting of 4 bouts of 30-seconds, while performing DQS or IQS with 30s rest, does not influence RFD or GRF in an IMTP. However, DQS without vibration for 4 bouts of 30s demonstrated an increase in RFD compared to iVib, but no other conditions. This suggests that athletes and coaches who are in search of various methods of eliciting an acute increase in power should consider implementing this protocol. In addition, utilizing WBV does not negatively effect force or power production. Thus, this specific methodology could be conducted as a general warm-up. These results may also suggest that a stimulus similar to a dynamic movement should be used prior to a maximal isometric action. Further research is needed to determine an optimal protocol for explosive isometric exercise subsequent to a specific warm-up in conjunction with WBV. 


\section{References}

Adams, J. B., Edwards, D., Serravite, D. H., Bedient, A. M., Huntsman, E., Jacobs, K. A., . . Signorile, J. F. (2009). Optimal frequency, displacement, duration, and recovery patterns to maximize power output following acute wholebody vibration. $J$ Strength Cond Res, 23(1), 237-245.

Alikhajeh, Y., Ramezanpour, M. R., \& Moghaddam, A. (2011). The Effect of Different Warm-up Protocols on young Soccer Players' sprint. Procedia-Social and Behavioral Sciences, 30, 1588-1592.

Anderson, P., Landers, G., \& Wallman, K. (2014). Effect of warm-up on intermittent sprint performance. Res Sports Med, 22(1), 88-99.

Berning, J. M., Adams, K. J., DeBeliso, M., Sevene-Adams, P. G., Harris, C., \& Stamford, B. A. (2010). Effect of functional isometric squats on vertical jump in trained and untrained men. J Strength Cond Res, 24(9), $2285-2289$.

Bush, J. A., Blog, G. L., Kang, J., Faigenbaum, A. D., \& Ratamess, N. A. (2015). Effects of quadriceps strength after static and dynamic whole-body vibration exercise. J Strength Cond Res, 29(5), 1367-1377.

Cardinale, M., \& Bosco, C. (2003). The use of vibration as an exercise intervention. Exercise and sport sciences reviews, 31(1), 3-7.

Cazas, V. L., Brown, L. E., Coburn, J. W., Galpin, A. J., Tufano, J. J., Laporta, J. W., \& Du Bois, A. M. (2013). Influence of rest intervals after assisted jumping on bodyweight vertical jump performance. J Strength Cond Res, 27(1), 64-68.

Chmielewska, D., Piecha, M., Blaszczak, E., Krol, P., Smykla, A., \& Juras, G. (2014). The effect of a single session of whole-body vibration training in recreationally active men on the excitability of the central and peripheral nervous system. J Hum Kinet, 41, 89-98.

Christensen, B. K., \& Nordstrom, B. J. (2008). The effects of proprioceptive neuromuscular facilitation and dynamic stretching techniques on vertical jump performance. J Strength Cond Res, 22(6), 1826-1831.

Cilli, M., Gelen, E., Yildiz, S., Saglam, T., \& Camur, M. (2014). Acute effects of a resisted dynamic warm-up protocol on jumping performance. Biol Sport, 31(4), 277-282.

Comfort, P., Allen, M., \& Graham-Smith, P. (2011). Comparisons of peak ground reaction force and rate of force development during variations of the power clean. The Journal of Strength \& Conditioning Research, 25(5), 1235-1239.

Comfort, P., Jones, P. A., McMahon, J. J., \& Newton, R. (2015). Effect of knee and trunk angle on kinetic variables during the isometric midthigh pull: test-retest reliability. Int J Sports Physiol Perform, 10(1), 58-63.

Cormie, P., Deane, R. S., Triplett, N. T., \& McBride, J. M. (2006). Acute effects of whole-body vibration on muscle activity, strength, and power. J Strength Cond Res, 20(2), 257-261.

Dabbs, N.C., Brown, L. E., Coburn, J. W., Lynn, S. K., Biagini, M. S., \& Tran, T. T. (2010). Effect of whole-body vibration warm-up on bat speed in women softball players. J Strength Cond Res, 24(9), 2296-2299.

Dabbs, N.C., Munoz, C. X., Tran, T. T., Brown, L. E., \& Bottaro, M. (2011). Effect of different rest intervals after whole-body vibration on vertical jump performance. J Strength Cond Res, 25(3), 662-667.

Dabbs, N.C., Tran, T. T., Garner, J. C., \& Brown, L. E. (2012). A brief review: Using whole-body vibration to increase acute power and vertical jump performance. Strength \& Conditioning Journal, 34(5), 78-84.

Dallas, G., Kirialanis, P., \& Mellos, V. (2014). The acute effect of whole body vibration training on flexibility and explosive strength of young gymnasts. Biol Sport, 31(3), 233-237.

Frantz, T.L., \& Ruiz, M. D. (2011). Effects of dynamic warm-up on lower body explosiveness among collegiate baseball players. J Strength Cond Res, 25(11), 2985-2990.

García-López, D., Garatachea, N., Marín, P. J., Martín, T., \& Herrero, A. J. (2012). Acute effects of whole-body vibrations on balance, maximal force and perceived exertion: Vertical platform versus oscillating platform. European Journal of Sport Science, 12(5), 425-430.

Garner, J. C., Blackburn, T., Weimar, W., \& Campbell, B. (2008). Comparison of electromyographic activity during eccentrically versus concentrically loaded isometric contractions. J Electromyogr Kinesiol, 18(3), 466-471.

Gerakaki, M. E., Evangelidis, P. E., Tziortzis, S., \& Paradisis, G. P. (2013). Acute effects of dynamic whole body vibration in well trained track \& field sprinters. J Phys Ed Sport, 13(3), 270-277.

Haff, G. G., Carlock, J. M., Hartman, M. J., Kilgore, J. L., Kawamori, N., Jackson, J. R., Stone, M. H. (2005). Forcetime curve characteristics of dynamic and isometric muscle actions of elite women olympic weightlifters. $J$ Strength Cond Res, 19(4), 741-748.

Haff, G. G., Ruben, R. P., Lider, J., Twine, C., \& Cormie, P. (2015). A comparison of methods for determining the rate of force development during isometric midthigh clean pulls. J Strength Cond Res, 29(2), 386-395.

Hamada, T., Sale, D. G., MacDougall, J. D., \& Tarnopolsky, M. A. (2000). Postactivation potentiation, fiber type, and twitch contraction time in human knee extensor muscles. J Appl Physiol (1985), 88(6), 2131-2137. 
Holt, B. W., \& Lambourne, K. (2008). The impact of different warm-up protocols on vertical jump performance in male collegiate athletes. J Strength Cond Res, 22(1), 226-229.

Kawamori, N., Rossi, S. J., Justice, B. D., Haff, E. E., Pistilli, E. E., O'BRYANT, H. S., Haff, G. G. (2006). Peak force and rate of force development during isometric and dynamic mid-thigh clean pulls performed at various intensities. The Journal of Strength \& Conditioning Research, 20(3), 483-491.

Khamoui, A. V., Brown, L. E., Nguyen, D., Uribe, B. P., Coburn, J. W., Noffal, G. J., \& Tran, T. (2011). Relationship between force-time and velocity-time characteristics of dynamic and isometric muscle actions. J Strength Cond Res, 25(1), 198-204.

Kurt, C., Toksoz, I., \& Dindar, M. D. (2014). The effects of two different whole-body vibration frequencies on isometric strength, anaerobic performance, and rating of perceived exertion. Journal of Physical Education and Sport, 14(2), 306.

Lamont, H. S., Cramer, J. T., Bemben, D. A., Shehab, R. L., Anderson, M. A., \& Bemben, M. G. (2010). Effects of adding whole body vibration to squat training on isometric force/time characteristics. J Strength Cond Res, 24(1), 171183.

Lowery, R. P., Duncan, N. M., Loenneke, J. P., Sikorski, E. M., Naimo, M. A., Brown, L. E., Wilson, J. M. (2012). The effects of potentiating stimuli intensity under varying rest periods on vertical jump performance and power. $J$ Strength Cond Res, 26(12), 3320-3325.

Marin, P. J., Ferrero, C. M., Menendez, H., Martin, J., \& Herrero, A. J. (2013). Effects of whole-body vibration on muscle architecture, muscle strength, and balance in stroke patients: a randomized controlled trial. Am J Phys Med Rehabil, 92(10), 881-888.

Reiman, M. P., Peintner, A. M., Boehner, A. L., Cameron, C. N., Murphy, J. R., \& Carter, J. W. (2010). Effects of dynamic warm-up with and without a weighted vest on lower extremity power performance of high school male athletes. The Journal of Strength \& Conditioning Research, 24(12), 3387-3395.

Ribeiro, A. S., Romanzini, M., Schoenfeld, B. J., Souza, M. F., Avelar, A., \& Cyrino, E. S. (2014). Effect of different warm-up procedures on the performance of resistance training exercises. Percept Mot Skills, 119(1), 133-145.

Rittweger, J., Beller, G., \& Felsenberg, D. (2000). Acute physiological effects of exhaustive whole-body vibration exercise in man. Clin Physiol, 20(2), 134-142.

Ritzmann, R., Kramer, A., Gollhofer, A., \& Taube, W. (2013). The effect of whole body vibration on the H-reflex, the stretch reflex, and the short-latency response during hopping. Scand J Med Sci Sports, 23(3), 331-339.

Ronnestad, B. R., Holden, G., Samnoy, L. E., \& Paulsen, G. (2012). Acute effect of whole-body vibration on power, one-repetition maximum, and muscle activation in power lifters. J Strength Cond Res, 26(2), 531-539.

Thompsen, A. G., Kackley, T., Palumbo, M. A., \& Faigenbaum, A. D. (2007). Acute effects of different warm-up protocols with and without a weighted vest on jumping performance in athletic women. J Strength Cond Res, 21(1), 5256.

Thompson, P. D., Arena, R., Riebe, D., Pescatello, L. S., \& American College of Sports, M. (2013). ACSM's new preparticipation health screening recommendations from ACSM's guidelines for exercise testing and prescription, ninth edition. Curr Sports Med Rep, 12(4), 215-217.

Turner, A. P., Sanderson, M. F., \& Attwood, L. A. (2011). The acute effect of different frequencies of whole-body vibration on countermovement jump performance. J Strength Cond Res, 25(6), 1592-1597.

West, D. J., Owen, N. J., Jones, M. R., Bracken, R. M., Cook, C. J., Cunningham, D. J., Crewther, B. T. (2011). Relationships between force-time characteristics of the isometric midthigh pull and dynamic performance in professional rugby league players. The Journal of Strength \& Conditioning Research, 25(11), 3070-3075. 